\title{
Absent Cortical Venous Filling Is Associated with Aggravated Brain Edema in Acute Ischemic Stroke
}

\author{
(D) H. Xia, (D) H. Sun, (D) S. He, (D) M. Zhao, (D)W. Huang, (D) Z. Zhang, (D) Y. Xue, (DP. Fu, and (D). Chen
}

\begin{abstract}
BACKGROUND AND PURPOSE: Predicting malignant cerebral edema can help identify patients who may benefit from appropriate evidence-based interventions. We investigated whether absent cortical venous filling is associated with more pronounced early brain edema, which leads to malignant cerebral edema.
\end{abstract}

\begin{abstract}
MATERIALS AND METHODS: Patients with acute ischemic stroke caused by large-vessel occlusion in the MCA territory who presented between July 2017 and September 2019 to our hospital were included. Collateral filling was rated using the modified Tan scale on CTA, and good collaterals were defined as a score of 2-3. The Cortical Vein Opacification Score (COVES) was calculated, and absent cortical venous filling was defined as a score of 0 . Early brain edema was determined using net water uptake on baseline CT images. Malignant cerebral edema was defined as a midline shift of $\geq 5 \mathrm{~mm}$ on follow-up imaging or a massive cerebral swelling leading to decompressive hemicraniectomy or death. Multivariate linear and logistic regression models were performed to analyze data.
\end{abstract}

RESULTS: A total of 163 patients were included. Net water uptake was significantly higher in patients with absent than in those with favorable cortical venous filling ( $8.1 \%$ versus $4.2 \% ; P<.001)$. In the multivariable regression analysis, absent cortical venous filling ( $\beta=2.04 ; 95 \% \mathrm{Cl}, 0.75-3.32 ; P=.002)$ was significantly and independently associated with higher net water uptake. Absent cortical venous filling (OR, 14.68; 95\% Cl, 4.03-53.45; $P<.001)$ and higher net water uptake (OR, 1.29; 95\% Cl, 1.05-1.58; $P=.016)$ were significantly associated with increased likelihood of malignant cerebral edema.

CONCLUSIONS: Patients with absent cortical venous filling were associated with an increased early brain edema and a higher risk of malignant cerebral edema. These patients may be targeted for optimized adjuvant antiedematous treatment.

ABBREVIATIONS: COVES = Cortical Vein Opacification Score; MCE = malignant cerebral edema; NWU = net water uptake; AIS = acute ischemic stroke; $\mathrm{OIT}=$ onset to imaging time; ICAS $=$ intracranial atherosclerosis

M alignant cerebral edema (MCE) is a lethal complication mainly of large infarctions in the MCA territory. ${ }^{1}$ It usually occurs within the first 3 days after the onset of stroke and is characterized by an abrupt neurologic decline associated with massive swelling of the infarcted brain tissues. ${ }^{2}$ The subsequent cerebral herniation due to intracranial pressure increase is the main cause

Received July 17, 2020; accepted after revision December 01.

From the Department of Radiology (H.X.), Shaoxing People's Hospital (Shaoxing Hospital, Zhejiang University School of Medicine), Shaoxing, Zhejiang, China; and Department of Radiology (H.S., S.H., M.Z., W.H., Z.Z., Y.X., P.F., W.C.), The First Affiliated Hospital of Wenzhou Medical University, Wenzhou, Zhejiang, China.

This study was funded by the Zhenjiang Provincial Key Laboratory of Aging and Neurological Disorder Research (LH-001), and the Scientific Technology Planning Projects of Wenzhou, China (Y20170216).

Please address correspondence to Weijian Chen, MD, Department of Radiology, The First Affiliated Hospital of Wenzhou Medical University, Nanbai Xiang St, Ouhai District, Wenzhou, Zhejiang, 325000, China; e-mail: wyyycwj@163.com

- Indicates open access to non-subscribers at www.ajnr.org

http://dx.doi.org/10.3174/ajnr.A7039

of death, with a mortality rate approaching $80 \%$ with conservative treatment. ${ }^{3}$ Three randomized controlled trials provided evidence that early decompressive surgery is an efficient way to increase the likelihood of favorable functional outcomes and reduce the mortality rate. ${ }^{4}$ Therefore, early identification of patients at risk of MCE would facilitate timely selection of appropriate evidence-based interventions.

Net water uptake (NWU), a quantitative imaging biomarker used to estimate water uptake in ischemic stroke lesions, was first applied to distinguish stroke onset within and beyond 4.5 hours. ${ }^{5}$ Mounting evidence suggests the importance of NWU because it has been reported as a surrogate marker for developing MCE. ${ }^{6}$ Recently, the arterial collateral status has been observed as it relates to the early edema progression rate. ${ }^{7}$ However, the absence of cortical venous filling could potentially be a better indicator of aggravating brain edema than the arterial collateral status because the venous system is responsible for approximately $70 \%$ of the cerebral blood volume. ${ }^{8}$ In a case series including 14 
patients, cranial venous outflow abnormalities increased the rate of early fatal edema after MCA infarction. ${ }^{9}$ Nonetheless, studies on the role of the cortical veins in the development of MCE are limited, and it remains unclear whether absent cortical venous filling is an independent risk factor affecting NWU in patients with stroke.

Recently, the Cortical Vein Opacification Score (COVES) was introduced as a simple method to assess the cortical venous filling status on CTA. It well reflects the intracranial microcirculation function and can predict whether patients will benefit from endovascular treatment. ${ }^{10}$ Thus, the aim of our study was to investigate the relationship between absent cortical venous filling, as assessed using the COVES, and early brain edema, as assessed using NWU, and whether they can predict the occurrence of MCE. We hypothesized that absent cortical venous filling would lead to an increase in early brain edema, which in turn leads to MCE.

\section{MATERIALS AND METHODS}

Data supporting the findings of this study are available from the corresponding author on reasonable request.

\section{Patients}

We retrospectively reviewed the data of patients diagnosed with acute ischemic stroke (AIS) caused by large-vessel occlusion in the MCA territory in our institution from July 2017 to September 2019. All procedures performed in studies involving human participants were in accordance with the ethical standards of the institutional and national research committee and with the 1964 Helsinki declaration and its later amendments or comparable ethical standards. Written informed consent was obtained from each patient or an appropriate family member. The inclusion criteria were as follows: 1) known time window from symptom onset to admission imaging of $<8$ hours; 2) multimodal CT performed on admission, which included NCCT, CTP, and CTA; 3) AIS with complete occlusion of the distal internal carotid artery, MCA, or both, confirmed by conventional and dynamic CTA constructed from CTP; 4) follow-up CT acquired 24-72 hours after symptom onset; and 5) absence of intracranial hemorrhage, or pre-existing infarction. Baseline clinical characteristics and demographic information were recorded including age, sex, NIHSS score, onset to imaging time (OIT), IV administration of tPA, and endovascular treatment.

\section{Image Acquisition}

All patients underwent multimodal CT at admission with NCCT, CTP, and CTA performed on a 64-section CT scanner (Lightspeed VCT; GE Healthcare). First, NCCT was performed $(120 \mathrm{kV}$; $300 \mathrm{~mA}$; section thickness, $5 \mathrm{~mm}$ ) from the foramen magnum to the vertex to detect early ischemic changes and exclude intracranial hemorrhage. If appropriate, CTP $(8 \mathrm{~cm}$ in the $\mathrm{z}$-axis using the shuttle mode technique, $80 \mathrm{kV}, 375 \mathrm{~mA}, 0.4$ s/rotation, 6-s prescan delay after injection of contrast medium, 60-s total imaging duration) with a total of 352 images by 22 consecutive spiral acquisitions was performed immediately after NCCT. A total of $50 \mathrm{~mL}$ of iodinated contrast agent was administered at a flow rate of $5 \mathrm{~mL} / \mathrm{s}$ followed by a saline flush of $50 \mathrm{~mL}$ at a rate of $5 \mathrm{~mL} / \mathrm{s}$. Subsequently, helical
CTA (100 kV; $500 \mathrm{~mA}$; section thickness, $0.625 \mathrm{~mm})$ was performed with acquisition from the aortic arch to the vertex after administration of $40 \mathrm{~mL}$ of an iodinated contrast agent injected at a rate of $4.0 \mathrm{~mL} / \mathrm{s}$ followed by a $30-\mathrm{mL}$ saline flush.

\section{Image Analysis}

Imaging data were independently assessed by 2 radiologists with more than 3 years of experience in stroke imaging, who were blinded to the clinical status and outcome data. The observers were informed about the laterality of symptoms just before evaluation. Any disagreements between the 2 observers were resolved by a senior medical chief neuroradiologist.

The ASPECTS was used to detect early ischemic burden on NCCT images. ${ }^{11}$ Early ischemic burden was defined as loss of gray-white differentiation and tissue hypoattenuation using a narrow window and level settings to maximize the contrast between the normal and infarcted brain.

Collateral filling was rated using the modified Tan scale on baseline CTA maximum intensity projection images as follows: 0 indicated absent collaterals of the occluded MCA territory, 1 indicated collaterals filling $>0 \%$ but $<50 \%$ of the occluded MCA territory, 2 indicated filling $>50 \%$ but $<100 \%$ of the occluded MCA territory, and 3 indicated $100 \%$ collateral filling of the occluded MCA territory. Poor collateral filling was defined as a score of 0 1 and good collateral filling as a score of $2-3 .^{12}$

The COVES was used to assess the venous opacification on baseline CTA source images, as absent (0), moderate (1), or full (2) in the vein of Labbe, sphenoparietal sinus, and superficial middle cerebral vein. The sum for the hemisphere ipsilateral to the occlusion was calculated and ranged between 0 and 6 . The absence of cortical vein opacification was defined as a score of 0 , and a score $>0$ was considered favorable as mentioned in a previous study. ${ }^{10}$

The ischemic lesion NWU was measured by a commercial software, Mistar (Apollo Medical Imaging Technology), using the singular value deconvolution with delay and dispersion method. First, previous thresholds were applied to measure the entire ischemic lesion (delay time $>3 \mathrm{~s}$ ) and ischemic core (relative cerebral blood flow $<30 \%) .{ }^{13}$ Next, the ROI was generated automatically in the ischemic lesion core. Then the core-based ROI was copied to the corresponding NCCT image using Mistar software. The ROI sampling of the ischemic core in NCCT was assessed by densitometric measurements and designated as $\mathrm{D}_{\text {ischemic }}$. A mirrored ROI was placed in the contralateral hemisphere within the normal tissue designated as $\mathrm{D}_{\text {normal }}$. To exclude the voxels of adjacent CSF and skull, a threshold value of 20-80 HU was chosen. $D_{\text {ischemic }}$ and $D_{\text {normal }}$ were then used to calculate the quantitative NWU based on the formula from Broocks et $\mathrm{al}^{14}$ as shown in Fig 1.

According to previous studies, ${ }^{15,16}$ MCE was defined as 1) a midline shift of $\geq 5 \mathrm{~mm}$ at the level of septum pellucidum or 2) a massive cerebral swelling requiring decompressive hemicraniectomy or leading to death.

\section{Statistical Analysis}

All statistical analyses were performed using SPSS 25.0 (IBM) and GraphPad Prism (GraphPad Software). Continuous variables were reported as means \pm SD or median (25th-75th percentile). 


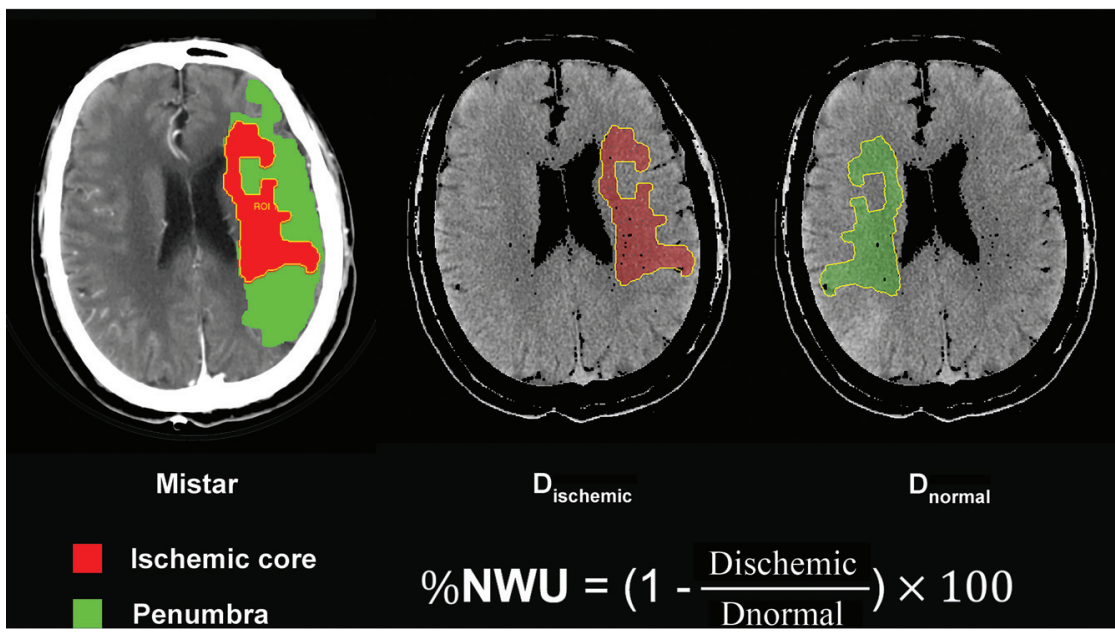

FIG 1. Quantification of NWU. NWU is determined in admission noncontrast CT. The ROI is generated automatically in the ischemic lesion core, which is measured with Mistar. The mean attenuation of $D_{\text {ischemic }}$ and $D_{\text {nornal }}$ are used to calculate NWU based on the formula shown.

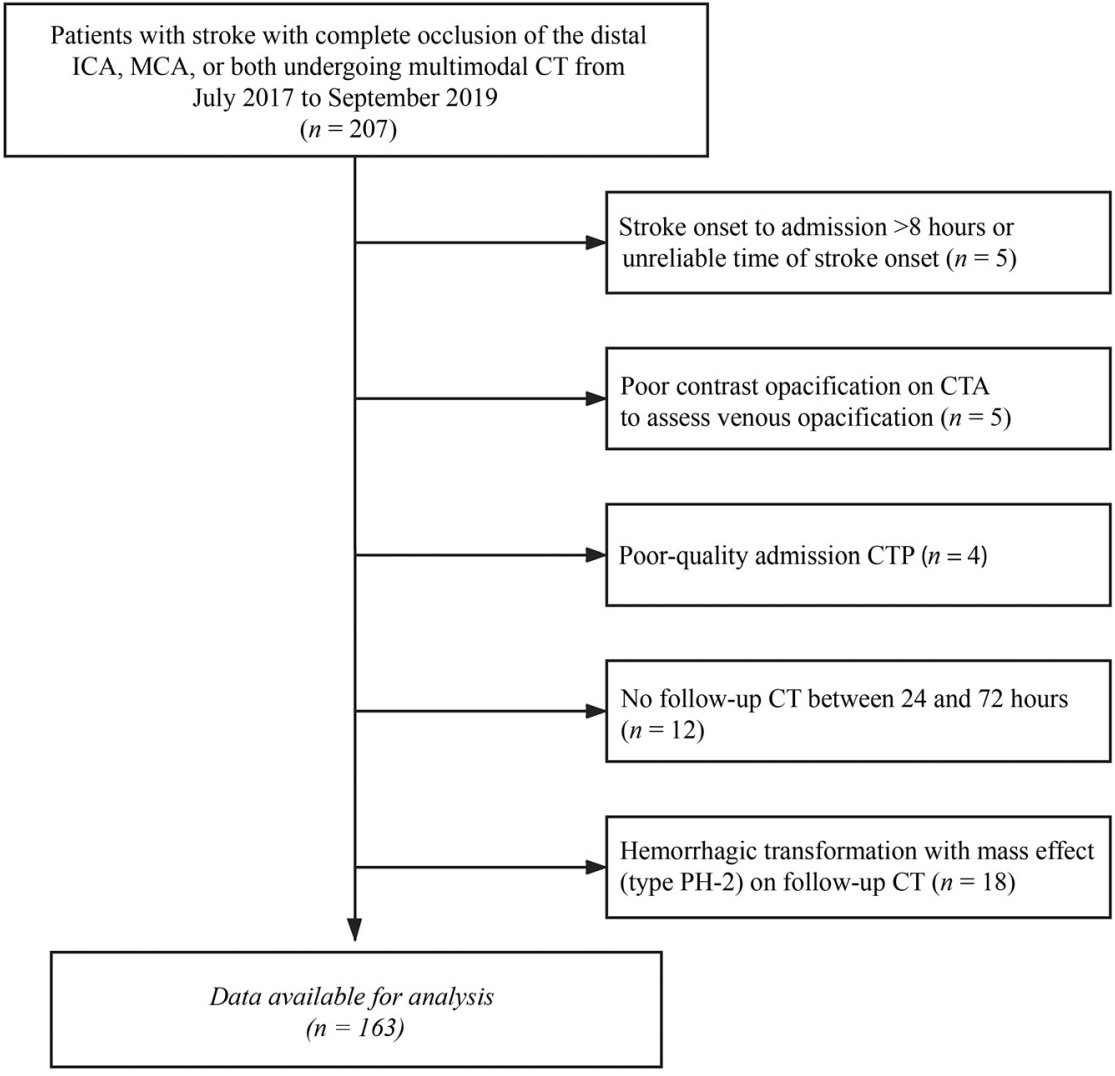

FIG 2. Flowchart of patient selection.

Categoric variables were reported as numbers (percentages). The differences among groups were evaluated using the Student $t$ test for normally distributed continuous data, Mann-Whitney $U$ test for non-normally distributed continuous data, and Pearson $\chi^{2}$ test for categoric data. The correlation between collateral filling, cortical venous filling status, and NWU was described by Kendall correlation analysis. The relationship between the cortical venous filling status (COVES was examined as a continuous variable and dichotomized into "absent" and "favorable" cortical venous filling) and early brain edema (NWU on NCCT images) was analyzed using linear regression. Variables with $P<.1$ in univariable linear regression were included in a multivariable linear regression analysis. Independent factors for MCE were evaluated using a binary logistic regression analysis. Factors with $P<.1$ from the univariate analysis were entered into the multivariable logistic regression analysis. Interrater reliability of the COVES score was tested by $\kappa$ statistics. Results with $P$ values of $<.05$ are considered significant for all statistical analyses.

\section{RESULTS}

During the study period, data on 207 consecutive patients with complete occlusion of the distal internal carotid artery, MCA, or both, undergoing multimodal CT were collected. A total of 163 patients met all inclusion criteria and were included in the analysis. The patient selection process is shown in Fig 2. The patient characteristics are listed in Table 1. The median age was 69 (IQR 58-78) years, and 103 (63\%) patients were men. The baseline NIHSS score was $11.34 \pm 5.43$, and the median OIT was 191 (IQR 116-259) minutes. Among all patients, 82 (50\%) underwent endovascular treatment, and 68 (42\%) received IV thrombolysis. There were 112 (69\%) patients with good collateral filling. At baseline, NWU was 4.9\% (IQR 2.98-7.33).

Comparing patients with absent $($ COVES $=0)$ versus favorable cortical venous filling (COVES $>0$ ), the former had a significantly higher baseline NIHSS score (13.5 versus 11; $P=.001$ ), lower ASPECTS (4.5 versus $9 ; P<.001$ ), and a lower rate of good collateral filling $(22 \%$ versus $80 \% ; P<.001)$. No significant differences were found in age, sex, OIT, endovascular treatment, or IV thrombolysis (Table 2).

Absent cortical venous filling correlated positively with NWU (Kendall $\tau=0.37$; 95\% CI, 0.27-0.46; $P<.001$ ). The collateral filling and NWU also showed a significant correlation (Kendall $\tau$ $=-0.30 ; 95 \% \mathrm{CI},-0.41$ to $-0.19 ; P<.001)$. Moreover, cortical 
Table 1: Patient characteristics according to the presence of MCE

\begin{tabular}{|c|c|c|c|c|}
\hline & & $\operatorname{MCE}(n=39)$ & Non-MCE $(n=124)$ & \\
\hline Patient Characteristic & Overall ( $n=163)$ & $24 \%$ & $76 \%$ & $P$ Value \\
\hline Age in years, median (IQR) & $69(58-78)$ & $74(61-81)$ & 69 (57.25-76) & .131 \\
\hline Male sex, $n(\%)$ & $103(63 \%)$ & $20(51 \%)$ & $83(67 \%)$ & .077 \\
\hline Admission NIHSS, mean \pm SD & $11.34 \pm 5.43$ & $14.03 \pm 5.11$ & $10.49 \pm 5.27$ & $<.001$ \\
\hline OIT in minutes, median (IQR) & 191 (116-259) & 201 (128-277) & $189.5(115.25-258.75)$ & .858 \\
\hline IV thrombolysis, $n$ (\%) & $68(42 \%)$ & $12(31 \%)$ & $56(45 \%)$ & .112 \\
\hline Endovascular treatment, $n(\%)$ & $82(50 \%)$ & $19(49 \%)$ & $63(51 \%)$ & .820 \\
\hline Good collateral filling, $n(\%)$ & $112(69 \%)$ & $12(31 \%)$ & $100(81 \%)$ & $<.001$ \\
\hline ASPECTS, median (IQR) & $9(7-10)$ & $4(2-7)$ & $9(8-10)$ & $<.001$ \\
\hline COVES $=0, n(\%)$ & $32(20 \%)$ & $26(67 \%)$ & $6(5 \%)$ & $<.001$ \\
\hline NWU in \%, median (IQR) & $4.89(2.98-7.33)$ & $8.01(6.29-11.19)$ & $4.15(2.59-6.11)$ & $<.001$ \\
\hline
\end{tabular}

Table 2: Patient characteristics according to the COVES

\begin{tabular}{|c|c|c|c|}
\hline & COVES $>0(n=131)$ & COVES $=0(n=32)$ & \\
\hline Patient Characteristic & $80 \%$ & $20 \%$ & $P$ Value \\
\hline Age in years, median (IQR) & 70 (60-77) & $68.5(53.75-78.75)$ & .383 \\
\hline Male sex, $n(\%)$ & $84(64 \%)$ & $19(60 \%)$ & .618 \\
\hline Admission NIHSS, median (IQR) & $11(6-14)$ & $13.5(10-18)$ & .001 \\
\hline OIT in minutes, median (IQR) & $188(118-261)$ & $205(110.5-252.75)$ & .910 \\
\hline IV thrombolysis, $n(\%)$ & $58(44 \%)$ & $10(31 \%)$ & .180 \\
\hline Endovascular treatment, $n(\%)$ & $65(50 \%)$ & $17(53 \%)$ & .722 \\
\hline Good collateral filling, $n(\%)$ & $105(80 \%)$ & $7(22 \%)$ & $<.001$ \\
\hline ASPECTS, median (IQR) & $9(7-10)$ & $4.5(2.25-7.75)$ & $<.001$ \\
\hline NWU in \%, median (IQR) & $4.24(2.73-6.46)$ & $8.11(6.08-11.00)$ & $<.001$ \\
\hline
\end{tabular}

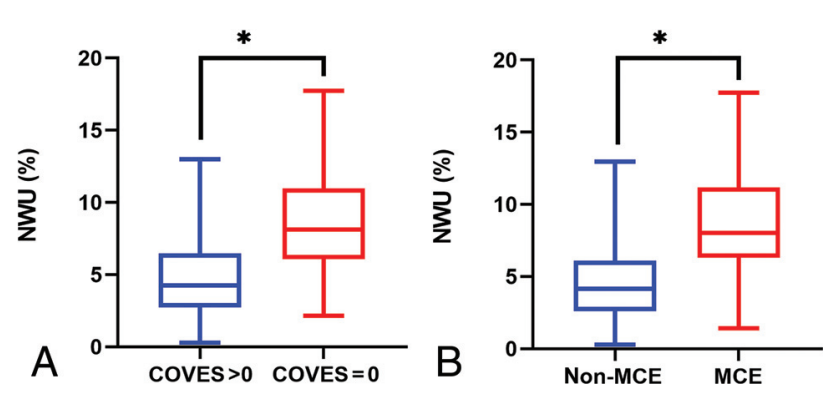

FIG 3. Relationship of cortical venous filling, early brain edema, and MCE. A, Boxplot shows that NWU was significantly higher in patients with COVES $=0$ than in those with COVES $>0 . B$, Boxplot shows that NWU was significantly higher in patients with MCE than in those without MCE. * indicates $P<.001$.

venous filling significantly correlated with collateral filling (Kendall $\tau=-0.50$; 95\% CI, -0.64 to $-0.34 ; P<.001$ ).

\section{Impact of Cortical Venous Filling on Early Brain Edema}

NWU was significantly higher in patients with absent cortical venous filling than in those with favorable cortical venous filling (8.1\% versus $4.2 \% ; P<.001$ ) (Fig 3). Furthermore, as seen in Table 2 and Table 3, in the multivariable linear regression analyses, the ASPECTS $(\beta=-0.50 ; 95 \% \mathrm{CI},-0.70$ to -0.30 ; $P<.001)$ and absent cortical venous filing ( $\beta=2.04$; 95\% CI, -0.75 to $3.32 ; P=.002$ ) were 2 significant factors associated with NWU adjusted for baseline NIHSS score and collateral filling (Table 3 and Fig 4).

\section{Factors Associated with MCE}

A total of 39 (24\%) patients developed MCE. As shown in Table 1, patients with MCE had higher baseline NIHSS score (14.03 versus $10.49 ; P<.001)$ and NWU $(8.0 \%$ versus $4.2 \% ; P<.001)$ (Fig 3), lower ASPECTS (4 versus 9; $P<.001$ ), higher rate of absent cortical venous filling (67\% versus $4.8 \% ; P<.001$ ), and lower rate of good collateral filling $(31 \%$ versus $81 \%, P<.001)$ compared with patients without MCE.

In the univariable logistic regression analysis, multiple factors were associated with MCE. The significant variables from the univariate analysis were entered into the multivariate logistic regression. Male sex (OR, 0.31; 95\% CI, 0.096-0.987; $P=.047$ ), ASPECTS (OR, 0.77; 95\% CI, 0.60-0.97; $P=.029$ ), absent cortical venous filling (OR, 14.68; 95\% CI, 4.03-53.44; $P<.001$ ), and NWU (OR, 1.29; 95\% CI, 1.05-1.58; $P=.016$ ) were significantly associated with increased likelihood of MCE after adjustment for confounding factors (Table 3).

\section{Interobserver Analysis}

Interrater reliability for COVES score on baseline CTA source images was substantial $(\kappa=0.75)$.

\section{DISCUSSION}

The aim of our study was to investigate the relationship between absent cortical venous filling and early brain edema assessed using NWU and its impact on the occurrence of MCE. Our study revealed that absent cortical venous filling was strongly associated with higher early brain edema, and both absent cortical venous filling and higher NWU were independent predictors of the risk for the development of MCE in patients with large-vessel occlusion in the MCA territory.

These results may support our original hypothesis that absent cortical venous filling is responsible for the onset of MCE by inducing early brain edema. The underlying pathophysiological mechanisms of MCE may be abnormal venous drainage; the 
Table 3: Univariable and multivariable linear regression analyses to predict NWU and univariable and multivariable logistic regression analyses to predict MCE

\begin{tabular}{|c|c|c|c|}
\hline \multicolumn{4}{|c|}{ Impact on NWU in multivariate regression } \\
\hline Parameter & Coefficient & $95 \% \mathrm{Cl}$ & $P$ Value \\
\hline \multicolumn{4}{|l|}{ Univariable analysis } \\
\hline Age (1-year increase) & 0.009 & $-0.03-0.05$ & .667 \\
\hline Male sex & -0.29 & $-1.38-0.79$ & .597 \\
\hline Admission NIHSS & 0.17 & $0.07-0.26$ & .001 \\
\hline Onset to imaging time (1 minute) & 0.004 & $-0.001-0.009$ & .147 \\
\hline Good collateral filling & -2.90 & $-3.93-1.86$ & $<.001$ \\
\hline ASPECTS & -0.70 & $-0.86-0.54$ & $<.001$ \\
\hline COVES $=0$ & 3.99 & $2.83-5.15$ & $<.001$ \\
\hline \multicolumn{4}{|l|}{ Multivariable analysis } \\
\hline Admission NIHSS & -0.01 & $-0.08-0.10$ & .811 \\
\hline Good collateral filling & -0.46 & $-1.59-0.67$ & .423 \\
\hline ASPECTS & -0.50 & $-0.70-0.30$ & $<.001$ \\
\hline COVES $=0$ & 2.04 & $0.75-3.32$ & .002 \\
\hline \multicolumn{4}{|l|}{ Prediction of MCE } \\
\hline \multicolumn{4}{|l|}{ Parameter } \\
\hline \multicolumn{4}{|l|}{ Univariable analysis } \\
\hline Age (1-year increase) & 1.02 & 0.99-1.05 & .300 \\
\hline Male sex & 0.52 & $0.25-1.08$ & .079 \\
\hline Admission NIHSS & 1.14 & $1.06-1.23$ & .001 \\
\hline OIT (1 minute) & 1.001 & 0.997-1.004 & .733 \\
\hline IV thrombolysis & 0.54 & $0.25-1.16$ & .115 \\
\hline Endovascular treatment & 0.92 & $0.45-1.89$ & .820 \\
\hline Good collateral filling & 0.11 & $0.05-0.24$ & $<.001$ \\
\hline ASPECTS & 0.59 & $0.50-0.70$ & $<.001$ \\
\hline COVES $=0$ & 39.33 & $13.68-113.12$ & $<.001$ \\
\hline NWU & 1.58 & $1.35-1.85$ & $<.001$ \\
\hline \multicolumn{4}{|l|}{ Multivariable analysis } \\
\hline Male sex & 0.31 & $0.10-0.99$ & .047 \\
\hline Admission NIHSS & 1.02 & $0.91-1.35$ & .730 \\
\hline Good collateral filling & 0.75 & $0.22-2.63$ & .657 \\
\hline ASPECTS & 0.77 & $0.60-0.97$ & .029 \\
\hline COVES $=0$ & 14.68 & $4.03-53.45$ & $<.001$ \\
\hline NWU & 1.29 & $1.05-1.58$ & .016 \\
\hline
\end{tabular}

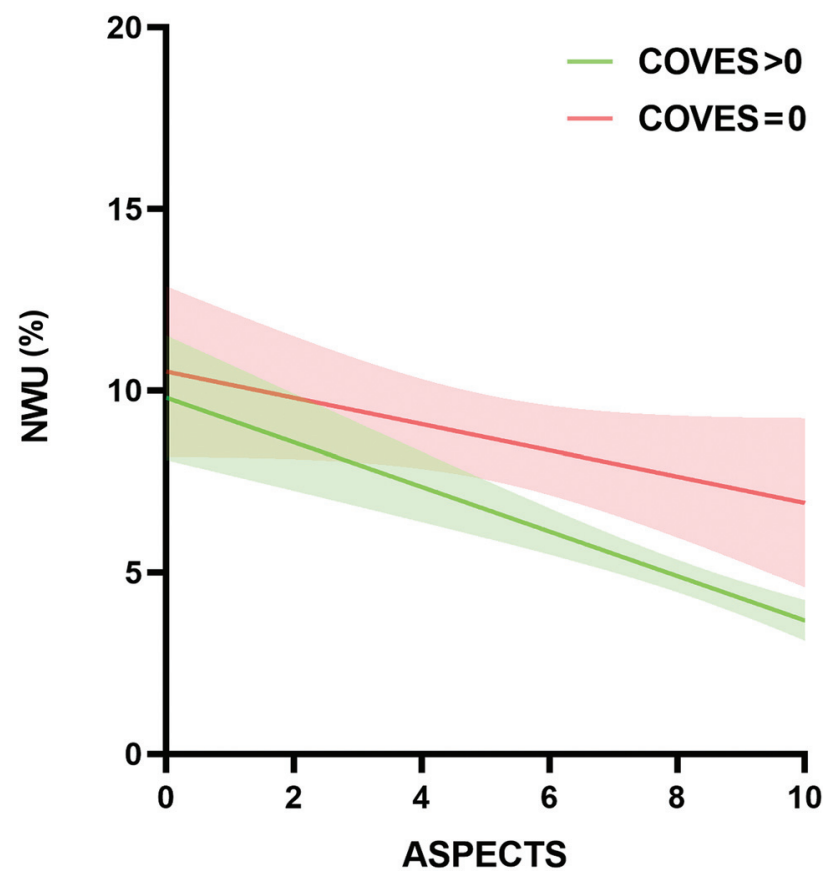

FIG 4. Impact of absent cortical venous filling on early brain edema according to baseline ASPECTS. subsequent resistance to CSF absorption would lead to increased venous pressure, which would increase the leakage of fluid into the perivascular space, leading to brain edema. ${ }^{17}$ Our results are in accordance with those of a recent study that investigated the association between quantitative lesion water uptake and malignant infarction. ${ }^{6}$ The authors concluded that NWU is a significant predictor for developing MCE. We further found that absent cortical venous filling was significantly associated with higher early brain edema and could predict the occurrence of MCE. Thus, these findings can be of great clinical significance in patients without cortical venous filling and can indicate who should receive adjuvant treatment with neuroprotectants and edema prevention therapies such as IV glibenclamide. ${ }^{18}$ However, whether absent cortical venous filling plays any other role in the development of MCE needs to be further elucidated.

Previous studies showed that collateral filling is significantly associated with an early edema progression rate and plays a role in the development of MCE. ${ }^{7,19}$ Our univariate analysis showed that patients with MCE had a significantly lower rate of good collateral filling than those without MCE. However, in the multivariate analysis, this factor could not predict NWU or the occurrence of MCE. This may be due to the high correlation between cortical venous filling and collateral filling (Kendall $\tau=-0.499 ; 95 \%$ CI, -0.638 to $-0.342 ; P<.001)$; however, no collinearity was found 
between them. A previous nonhuman primate model showed that absent cortical venous filling indicated a less extensive collateral circulation. $^{20} \mathrm{~A}$ recent clinical study that included 228 patients with AIS also demonstrated that venous outflow is affected by the reduction of arterial flow. ${ }^{21}$ Therefore, this suggests that cortical venous filling can provide additional information on both the occurrence of MCE and arterial collateral status. From our results, we can reasonably infer that cortical veins may play a more important role than arterial collateral in the development of MCE. These findings prompt us to combine arterial collateral and cortical vein to better evaluate patients with stroke, resulting in better treatment.

Some clinical factors may also predict MCE. Previous studies in Western countries showed that younger age significantly increased the risk of MCE. ${ }^{22,23}$ The possible reason for this is that the brain volume gradually decreases with increasing age; thus, younger patients are more likely to develop MCE because there is a lower intracranial volume to accommodate brain edema. ${ }^{1}$ However, the results of our study did not show the same findings. This discrepancy may be attributed to the differences in the etiologic composition of patients with stroke between China and Western countries. Intracranial atherosclerosis (ICAS) is common in the Chinese population, accounting for $33 \%-50 \%$ of AIS cases. ${ }^{24}$ In contrast, AIS is most commonly caused by cardioembolism in Western countries. Previous clinical research showed patients with cardioembolism tend to be older than those with ICAS $^{25}$ and are more likely to experience a large hemispheric infarction, ${ }^{26}$ which is the main cause of MCE. ${ }^{27}$ Our patients may have a higher proportion of ICAS, which makes the effect of age on MCE insignificant. However, our study did not measure the proportions of each etiology and the role of etiology in age and MCE because this was not the primary objective of this study. Another clinical factor, female sex, was shown to be an independent predictor of MCE, which is consistent with the results of previous studies ${ }^{28,29}$ indicating that a more efficacious stroke management may be needed in female patients. Although research efforts with a focus on sex differences in neuroinflammation, neuroprotection, and neuronal cell death signaling have recently received more attention, ${ }^{30-32}$ the reasons for this phenomenon remain obscure.

To the best of our knowledge, this is the first study to investigate the relationship between cortical venous filling, early brain edema, and MCE. One of the strengths of our study is that early brain edema was directly assessed by NWU, which calculates the water uptake based on the CT attenuation and has been proved as the criterion standard for histopathologic water uptake volume measurement. ${ }^{33,34}$ In addition, we selected the COVES to evaluate intracranial venous filling. This scoring method removes the deep cerebral venous system and more accurately reflects the status of the microcirculation. ${ }^{10,35}$ Although dynamic CTA may be a better way to assess cortical venous filling than conventional $\mathrm{CTA},{ }^{36}$ this technique requires advanced imaging and complex postprocessing. Our method was simple and convenient to use and has achieved good interobserver agreement, as previously described. ${ }^{10}$

This study has several limitations. First, it was a retrospective single-center study, and the number of patients was relatively small; thus, selection bias inevitably existed. Another drawback of this study can be attributed to the large variation in venous anatomy, though the 3 cortical veins chosen for the COVES had lesser anatomic variability than that of other veins. Third, NWU measurement may be imprecise in very small ischemic core volumes. However, we improved the precision using the Mistar software to measure the ischemic core assisting in determining the ROI. Furthermore, the recanalization status is a significant predictor of the occurrence of MCE. However, in this study, we did not include this factor mainly because many patients without endovascular treatment were unable to obtain recanalization data because of the absence of follow-up vascular imaging. Finally, we could not determine whether absent cortical venous filling leads to MCE because of early brain edema; we aim to perform a mediation analysis to explore this hypothesis more thoroughly in future studies.

\section{CONCLUSIONS}

We observed that absent cortical venous filling was significantly associated with early brain edema and the occurrence of MCE. Early brain edema may be the link between absent cortical venous filling and MCE. The clinical implication of these results is that absent cortical venous filling may assist in the selection of patients at risk for MCE in whom optimized adjuvant antiedematous treatment would be indicated.

Disclosures: Weijian Chen—RELATED: Grant: Zhenjiang Provincial Key Laboratory of Aging and Neurological Disorder Research (LH-001), the Scientific Technology Planning Projects of Wenzhou and China (Y20170216).

\section{REFERENCES}

1. Hacke W, Schwab S, Horn M, et al. "Malignant" middle cerebral artery territory infarction: clinical course and prognostic signs. Arch Neurol 1996;53:309-15 CrossRef Medline

2. Qureshi AI, Suarez JI, Yahia AM, et al. Timing of neurologic deterioration in massive middle cerebral artery infarction: a multicenter review. Crit Care Med 2003;31:272-77 CrossRef Medline

3. Berrouschot J, Sterker M, Bettin S, et al. Mortality of space-occupying (“malignant") middle cerebral artery infarction under conservative intensive care. Intensive Care Med 1998;24:620-23 CrossRef Medline

4. Vahedi K, Hofmeijer J, Juettler E, et al. Early decompressive surgery in malignant infarction of the middle cerebral artery: a pooled analysis of three randomised controlled trials. Lancet Neurol 2007;6:21522 CrossRef Medline

5. Minnerup J, Broocks G, Kalkoffen J, et al. Computed tomographybased quantification of lesion water uptake identifies patients within 4.5 hours of stroke onset: a multicenter observational study. Ann Neurol 2016;80:924-34 CrossRef Medline

6. Broocks G, Flottmann F, Scheibel A, et al. Quantitative lesion water uptake in acute stroke computed tomography is a predictor of malignant infarction. Stroke 2018;49:1906-12 CrossRef Medline

7. Broocks G, Kemmling A, Meyer L, et al. Computed tomography angiography collateral profile is directly linked to early edema progression rate in acute ischemic stroke. Stroke 2019;50:3424-30 CrossRef Medline

8. Ito H, Kanno I, Iida H, et al. Arterial fraction of cerebral blood volume in humans measured by positron emission tomography. Ann Nucl Med 2001;15:111-16 CrossRef Medline

9. Yu W, Rives J, Welch B, et al. Hypoplasia or occlusion of the ipsilateral cranial venous drainage is associated with early fatal edema of middle cerebral artery infarction. Stroke 2009;40:3736-39 CrossRef Medline 
10. Jansen IGH, van Vuuren AB, van Zwam WH, et al. Absence of cortical vein opacification is associated with lack of intra-arterial therapy benefit in stroke. Radiology 2018;286:643-50 CrossRef Medline

11. Pexman JH, Barber PA, Hill MD, et al. Use of the Alberta Stroke Program Early CT Score (ASPECTS) for assessing CT scans in patients with acute stroke. AJNR Am J Neuroradiol 2001;22:1534-42 Medline

12. Tan IY, Demchuk AM, Hopyan J, et al. CT angiography clot burden score and collateral score: correlation with clinical and radiologic outcomes in acute middle cerebral artery infarct. AJNR Am J Neuroradiol 2009;30:525-31 CrossRef Medline

13. Lin L, Bivard A, Kleinig T, et al. Correction for delay and dispersion results in more accurate cerebral blood flow ischemic core measurement in acute stroke. Stroke 2018;49:924-30 CrossRef Medline

14. Broocks G, Flottmann F, Ernst M, et al. Computed tomographybased imaging of voxel-wise lesion water uptake in ischemic brain: relationship between density and direct volumetry. Invest Radiol 2018;53:207-13 CrossRef Medline

15. Cheng Y, Wu S, Wang Y, et al. External validation and modification of the edema score for predicting malignant brain edema after acute ischemic stroke. Neurocrit Care 2020;32:104-12 CrossRef Medline

16. Kauw F, Bennink E, de Jong $\mathrm{H}$, et al. Intracranial cerebrospinal fluid volume as a predictor of malignant middle cerebral artery infarction. Stroke 2019; May 16 [Epub ahead of print] CrossRef Medline

17. Alperin N, Lee SH, Mazda M, et al. Evidence for the importance of extracranial venous flow in patients with idiopathic intracranial hypertension (IIH). Acta Neurochir Suppl 2005;95:129-32 CrossRef Medline

18. Vorasayan P, Bevers MB, Beslow LA, et al. Intravenous glibenclamide reduces lesional water uptake in large hemispheric infarction. Stroke 2019;50:3021-27 CrossRef Medline

19. Horsch AD, Dankbaar JW, Stemerdink TA, et al. Imaging findings associated with space-occupying edema in patients with large middle cerebral artery infarcts. AJNR Am J Neuroradiol 2016;37:831-37 CrossRef Medline

20. Sasaki M, Honmou O, Radtke C, et al. Development of a middle cerebral artery occlusion model in the nonhuman primate and a safety study of I.V. infusion of human mesenchymal stem cells. PloS One 2011;6:e26577 CrossRef Medline

21. Zhang S, Lai Y, Ding X, et al. Absent filling of ipsilateral superficial middle cerebral vein is associated with poor outcome after reperfusion therapy. Stroke 2017;48:907-14 CrossRef Medline

22. Miao J, Song X, Sun W, et al. Predictors of malignant cerebral edema in cerebral artery infarction: a meta-analysis. J Neurol Sci 2020;409:116607 CrossRef Medline
23. Wu S, Yuan R, Wang Y, et al. Early prediction of malignant brain edema after ischemic stroke. Stroke 2018;49:2918-27 CrossRef Medline

24. Wang Y, Zhao X, Liu L, et al. Prevalence and outcomes of symptomatic intracranial large artery stenoses and occlusions in China: the Chinese Intracranial Atherosclerosis (CICAS) Study. Stroke 2014;45:663-69 CrossRef Medline

25. Lee JS, Hong JM, Lee KS, et al. Endovascular therapy of cerebral arterial occlusions: intracranial atherosclerosis versus embolism. J Stroke Cerebrovasc Dis 2015;24:2074-80 CrossRef Medline

26. Rebello LC, Bouslama M, Haussen DC, et al. Stroke etiology and collaterals: atheroembolic strokes have greater collateral recruitment than cardioembolic strokes. Eur J Neurol 2017;24:762-67 CrossRef Medline

27. Wartenberg KE. Malignant middle cerebral artery infarction. Curr Opin Crit Care 2012;18:152-63 CrossRef Medline

28. Kasner SE, Demchuk AM, Berrouschot J, et al. Predictors of fatal brain edema in massive hemispheric ischemic stroke. Stroke 2001;32:2117-23 CrossRef Medline

29. Jaramillo A, Góngora-Rivera F, Labreuche J, et al. Predictors for malignant middle cerebral artery infarctions: a postmortem analysis. Neurology 2006;66:815-20 CrossRef Medline

30. Klein SL, Marriott I, Fish EN. Sex-based differences in immune function and responses to vaccination. Trans $\mathrm{R}$ Soc Trop Med Hyg 2015;109:9-15 CrossRef Medline

31. Mirza MA, Ritzel R, Xu Y, et al. Sexually dimorphic outcomes and inflammatory responses in hypoxic-ischemic encephalopathy. $J$ Neuroinflammation 2015;12:32 CrossRef Medline

32. Spychala MS, Honarpisheh P, McCullough LD. Sex differences in neuroinflammation and neuroprotection in ischemic stroke. $J$ Neurosci Res 2017;95:462-71 CrossRef Medline

33. Broocks G, Flottmann F, Ernst M, et al. Computed tomographybased imaging of voxel-wise lesion water uptake in ischemic brain: relationship between density and direct volumetry. Invest Radiol 2018;53:207-13 CrossRef

34. Dzialowski I, Klotz E, Goericke S, et al. Ischemic brain tissue water content: CT monitoring during middle cerebral artery occlusion and reperfusion in rats. Radiology 2007;243:720-26 CrossRef Medline

35. Hoffman H, Ziechmann R, Swarnkar A, et al. Cortical vein opacification for risk stratification in anterior circulation endovascular thrombectomy. J Stroke Cerebrovasc Dis 2019;28:1710-17 CrossRef Medline

36. van den Wijngaard IR, Wermer MJ, Boiten J, et al. Cortical venous filling on dynamic computed tomographic angiography: a novel predictor of clinical outcome in patients with acute middle cerebral artery. Stroke 2016;47:762-67 CrossRef 